\title{
Access to penile prostheses differ across provinces in Canada: A survey of Canadian urologists
}

\author{
Stewart Whalen ${ }^{1}$; Gavin Langille ${ }^{2}$; Josh White ${ }^{1}$; Greg Bailly ${ }^{1}$ \\ ${ }^{1}$ Department of Urology, Dalhousie University, Halifax, NS Canada; ${ }^{2}$ Department of Urology, Dalhousie University, \\ St. John, NB, Canada
}

Cite as: Whalen S, Langille G, White J, et al. Access to penile prostheses differ across provinces in Canada: A survey of Canadian urologists. Can Urol Assoc J 2020 December 15; Epub ahead of print. http://dx.doi.org/10.5489/cuaj.6867

Published online December 15, 2020

$* * *$

\section{Abstract}

Introduction: Surgical implantation of a penile prosthesis is the gold standard treatment for refractory erectile dysfunction. The purpose of this study was to investigate use of and access to these procedures in Canada.

Methods: Canadian urologists known to perform penile prostheses procedures were surveyed on areas such as surgical volume, type of device used, and the direct cost to patients for both malleable and inflatable devices.

Results: Of the 50 urologists invited to participate in the study, 34 (68\%) completed the online survey. Participants represented nine Canadian provinces and included a mix of academic $(65 \%)$ and community (35\%) urologists. Most participants (79\%) performed less than 10 procedures per year. Roughly three-quarters of participants (74\%) used inflatable devices in over $90 \%$ of cases, while half implanted inflatable devices exclusively. The most common devices implanted were American Medical Systems (AMS). Participants from Alberta, Manitoba, New Brunswick, and Newfoundland reported full coverage for both malleable and inflatable devices. Saskatchewan was the only province where no coverage was reported. The remaining provinces were found to have variable degrees of coverage. Across all centers without full coverage, the median reported cost to patients for a malleable and inflatable device was $\$ 5000$ and $\$ 6000$, respectively.

Conclusions: The urologists surveyed most commonly perform inflatable penile prostheses procedures. Significant geographical differences exist with respect to reported coverage for these procedures. This study highlights the need for continued advocacy on behalf of the urological community towards the goal of equity in coverage for penile prostheses across Canada. 


\section{Introduction}

Erectile dysfunction (ED) is a common clinical entity with prevalence in men over the age of 40 estimated to be $48.2 \%$ in Canada. ${ }^{1}$ ED significantly impacts the quality of life of men and their partners and is a well established risk factor for the development of depressive symptoms in those with the condition. ${ }^{2-3}$ Contemporary treatment follows a step wise approach beginning with phosphodiesterase type 5 (PDE5) inhibitors followed by second line options including vacuum erection devices and intracavernous or intraurethral injections of vasoactive agents. ${ }^{4}$ Despite the high success rates of non-surgical approaches, a subset of patients continue to suffer ED refractory to medical treatment. ${ }^{5}$ For this carefully selected patient population, surgical implantation of a penile prosthesis is an important treatment option.

Since their earliest description in the 1950s, a number of penile prostheses have been developed and used to treat ED. ${ }^{6}$ Modern devices consist of malleable (semi-rigid) and inflatable penile prostheses (IPPs). The type of device implanted depends on a number of factors including patient preference, surgeon experience, indication for procedure, the patient's manual dexterity, penile size and cognitive function. ${ }^{7}$ In Canada's publicly funded healthcare system, availability of government funding represents an additional factor when determining type of device implanted.

Three-piece IPPs are widely regarded as the gold standard implant and are the most common devices implanted today. ${ }^{8}$ IPPs have the advantage of allowing for both penile rigidity and flaccidity and have reported patient satisfaction rates as high as $97 \%$ for the American Medical Systems (AMS) 700CX. ${ }^{9}$ High rates of patient satisfaction appear to persist over time. Chierigo et al. found $60 \%$ of patients still using their IPP with high satisfaction at 20 year follow up. ${ }^{10}$ Malleable devices have the advantage of lower cost, technical ease and lower complication rates and remain an option in certain patient populations including those with spinal cord injuries. ${ }^{11}$ While overall patient satisfaction with malleable devices is lower than that of IPPs, it remains high, with rates reported between $71-75 \%$ for the AMS 600-650. ${ }^{9,12}$

The objective of this study was to evaluate access to and the direct cost to patients for penile prostheses in Canada as well as to determine practice patterns among Canadian urologists performing these procedures.

\section{Methods}

Participants were Canadian urologists who perform penile prostheses procedures. They were identified by industry partners who maintain databases of implanters. There were no further inclusion or exclusion criteria. Participants were invited to complete a web-based survey via email. One follow up email invitation was sent. Ethics approval was obtained with the Nova Scotia Health Authority Research Ethics Board (File \#1022258). Participation was voluntary and no compensation was provided. The survey consisted of ten questions including demographic information, case volume, type of device implanted and coverage available at the participant's 
centre. Responses were collected between May and June of 2017 and stored through the online survey platform Opinio®. Descriptive statistical analysis was employed.

\section{Results}

Of the 50 urologists invited to participate, 34 (68\%) completed the online survey. Results are outlined in Table 1. Participants were asked for responses to reflect their most recent year of practice. Participants represented 9 Canadian provinces with the greatest number of participants coming from the province of Ontario $(n=11,32.4 \%)$. The majority of participants $(n=22,64.7 \%)$ worked in an academic centre.

With respect to case volume, $79.4 \%(\mathrm{n}=27)$ of participants reported doing 10 or less cases per year. The greatest number of participants $(n=15,44.1 \%)$ reported case volume of between 5 to 10 cases per year. High volume surgeons with case volume of greater than 20 cases per year $(n=7,20.6 \%)$ worked in academic centres. Participants reported an overwhelming preference for IPPs with these being used in an average proportion of $87.4 \%$ of cases (range 15\%-100\%). Half of study participants reported using IPPs exclusively. AMS devices alone were used by $88.2 \%$ $(n=30)$ of participants while the remaining $11.8 \%(n=4)$ used both AMS and Coloplast devices.

The majority of participants $(n=23,67.6 \%)$ indicated that their centre provided full coverage for both malleable and IPPs with no out of pocket expense to patients. Of the remaining participants, $14.7 \%(n=5)$ reported full coverage for malleable and "the patient pays the difference in cost between IPP and malleable" for IPPs, $11.8 \%(n=4)$ reported no coverage for either device, $2.9 \%(\mathrm{n}=1)$ reported "other" coverage for both devices and 2.9\% $(\mathrm{n}=1)$ reported "other" coverage for malleable and "the patient pays the difference in cost between IPP and malleable" for IPPs.

If not fully covered at their centre, participants were asked to report the estimated direct out of pocket cost to patients. Of the 5 participants whose centre did not provide full coverage for malleable devices, the median estimated cost to patients was $\$ 5,000$ (range $\$ 2,000-\$ 8,000$ ). Of the 11 participants whose centre did not provide full coverage for IPPs, the median estimated cost to patients was $\$ 6,000$ (range $\$ 3,500-\$ 12,000$ ).

Reported coverage was similar between academic and community urologists with $66.7 \%$ $(\mathrm{n}=8)$ of community urologists and $68.2 \%(\mathrm{n}=15)$ of academic urologists reporting full coverage for both devices at their centre. IPPs were the most common devices used in both groups.

Academic and community urologists reported using IPPs in an average proportion of $84.2 \%$ and 92.9\% of cases respectively.

Reported coverage differed across provinces. All participants from the provinces of Alberta, Manitoba, New Brunswick and Newfoundland reported full coverage for both devices. The lone participant from Saskatchewan indicated no coverage for either device. Participants from Nova Scotia reported full coverage for malleable and "the patient pays the difference in cost between IPP and malleable" for IPPs. Participants from the remaining provinces of British 
Columbia, Ontario and Quebec reported variable coverage within their provinces. The majority of participants from Ontario $(54.5 \%, \mathrm{n}=6)$ and British Columbia $(75 \%, \mathrm{n}=6)$ reported full coverage for both devices while one of the two participants from Quebec reported full coverage for both devices.

IPPs were overwhelmingly the most common device implanted in every province with the exception of Nova Scotia. IPPs were reported to be used in $90 \%$ or greater of cases in provinces where participants indicated full coverage for both devices. This included an average proportion of 98.2\% of cases in Alberta, 97.5\% in New Brunswick, 100\% in Newfoundland and 90\% in Manitoba. The lone participant from Saskatchewan, where no coverage was reported, used IPPs exclusively. Provinces with variable coverage reported using IPPs in the majority of their cases with an average proportion of $88.1 \%$ of cases in British Columbia, $82.4 \%$ in Ontario and $82.5 \%$ in Quebec. Nova Scotia was a significant outlier with IPPs used in a reported average proportion of $15 \%$ of cases.

The breakdown between device implanted varied based on coverage reported by participants. Among participants who indicated full coverage for both devices $(n=23,67.6 \%)$, IPPs were used in an average proportion of $95.0 \%$ of cases. In contrast, IPP usage was on average $30.0 \%$ for participants who reported full coverage for malleable and "the patient pays the difference in cost between IPP and malleable" for IPPs $(n=5,14.7 \%)$. Those who indicated no coverage for either device $(\mathrm{n}=4,11.8 \%)$ used IPPs in an average proportion of $98.7 \%$ of cases. The remaining two participants, one of whom indicated "other" coverage for both devices and the other who indicated "other" coverage for malleable and "the patient pays the difference in cost between IPP and malleable" for IPPs, reported IPP use in 100\% and 85\% of cases, respectively.

\section{Discussion}

Our study found geographical differences in coverage for penile prostheses across provinces in Canada. Variable coverage based on participant's centre was reported within the largest provinces of British Columbia, Ontario and Quebec. Overall, 67.7\% of participants indicated full coverage for both malleable and IPPs at their centre. Among participants who reported less than full coverage, direct out of pocket cost to patients was found to be a median of $\$ 5,000$ and $\$ 6,000$ for malleable and IPPs respectively. The lone participant from Saskatchewan reported the highest out of pocket cost to patients at $\$ 8,000$ per malleable case and $\$ 12,000$ per IPP case. This demonstrates the high upfront costs of these procedures which can be passed along to patients where coverage doesn't exist. While initial costs may be seemingly high, penile prostheses have been shown to be the most cost-effective ED intervention following failed PDE5 treatment, supporting their use and coverage by health care centres and governments. ${ }^{13}$ 
IPPs are overwhelmingly the most common devices implanted today with their use having been documented in 89 to $96 \%$ of cases in the United States between the years 2003 and 2015. ${ }^{14}$ Further studies have shown the trend away from malleable and toward IPPs growing further. ${ }^{15}$ While our study highlights access issues in Canada, it does demonstrate a similar preference for IPPs among Canadian urologists surveyed. Overall, IPPs were reported as being used in an average proportion of $87.4 \%$ of cases. High rates of IPP use were observed among academic and community urologists surveyed as well as across all provinces with the exception of Nova Scotia.

A number of factors have been associated with implantation of an IPP vs. malleable device in the United States including higher surgeon volume and possession of commercial insurance. ${ }^{15}$ Our study found higher rates of IPP implantation (95.0 vs. $30.0 \%$ ) among surgeons with full coverage at their centre when compared to surgeons working at a centre in which malleable devices are covered and patients pay the difference in cost between IPPs and malleable for IPPs. This suggests that due to financial barriers patients may be opting for the device with no out of pocket cost and foregoing the standard of care IPP. This trend was most evident in the province of Nova Scotia where average out of pocket cost for an IPP was reported as $\$ 4250$ per case. Participants from this province used IPPs in an average proportion of only $15 \%$ of cases. Interestingly, participants who indicated no coverage for either device used IPPs at the highest overall proportion of $98.7 \%$ of cases. It is plausible that patients with the financial means to pay out of pocket to begin with are willing to pay the additional amount for the superior device.

The etiology of ED in individuals undergoing penile prostheses surgery is variable. However, a significant minority of patients seeking this intervention will have developed ED secondary to radical treatment of prostate cancer. A recent review of the literature by Bajic et al., found that roughly $20 \%$ of patients undergoing implantation of a penile prosthesis had pelvic surgery or trauma as the etiology of their ED while a further 5\% had ED secondary to nonsurgical management of prostate cancer. ${ }^{16}$ Patient satisfaction has been shown to be high in individuals undergoing implantation of a penile prosthesis following radical prostatectomy, highlighting the potential importance of this intervention in the post cancer recovery pathway. ${ }^{17-}$ 18

This study begs the question as to why many centres continue to charge patients either the full or partial cost of penile prostheses, when the majority of other prosthetic devices are fully covered by the Canadian health care system. It is proposed that the lack of coverage in many centres likely originates back to when these procedures were first introduced and ED was often still considered a psychogenic condition. As such, these procedures were considered more cosmetic rather than medically necessary interventions that lead to significant improvement in quality of life. With the acceptance in the past two decades that most cases of ED are related to organic and physical causes it is now recognized that $\mathrm{ED}$ is a medical condition that requires a 
combination of either medical or surgical treatment. Many would argue that the surgical implantation of a penile prosthesis is the gold standard treatment for ED and therefore should be fully funded.

To our knowledge, this is the first study investigating access to penile prostheses in Canada as well as practice patterns of Canadian urologists implanting these devices. The results identified the province of Nova Scotia as a significant outlier with respect to proportion of malleable vs. IPPs implanted, likely on the basis of cost. By demonstrating this inequity, we were able to successfully lobby for expanded IPP coverage in Nova Scotia.

Strengths of the study include a high response rate and geographically representative sample of participants. The study is limited by its survey methodology. Responses are subject to recall bias and largely represent estimates on behalf of the responding urologists. Further work is required including review of institutional data, billing information and patient surveys to more accurately characterize practice patterns and financial barriers to patients seeking penile prostheses procedures.

\section{Conclusions}

Our study demonstrates financial barriers to treatment and inequitable access to penile prostheses procedures across provinces in Canada. As healthcare costs continue to rise and governments look for ways to decrease spending it is imperative that the urologic community step up advocacy efforts to maintain existing coverage while making the case for expanded coverage where needed in order to ensure equitable access for patients. ED must be considered an important medical condition that has a significant impact on quality of life, and therefore penile prostheses procedures should be fully funded across all centres offering them in Canada. 


\section{References}

1. Grover SA, Lowensteyn I, Kaouache M, et al. The prevalence of erectile dysfunction in the primary care setting: Importance of risk factors for diabetes and vascular disease. Arch Intern Med 2006;166:213-9.

2. Shabsigh R, Klein LT, Seidman S, et al. Increased incidence of depressive symptoms in men with erectile dysfunction. Urology 1998;52.5:848-52.

3. Nelson CJ, Mulhall JP, Roth AJ, et al. The association between erectile dysfunction and depressive symptoms in men treated for prostate cancer. J Sex Med 2011;8:560-66.

4. Bella AJ, Lee JC, Carrier S, et al. 2015 CUA practice guidelines for erectile dysfunction. Can Urol Assoc J 2015;9:23-29.

5. Montorsi F, Rigatti P, Carmignani G, et al. AMS three-piece inflatable implants for erectile dysfunction: A long-term multi-institutional study in 200 consecutive patients. Eur Urol 2000;37:50-55.

6. Henry, GD. Historical review of penile prosthesis design and surgical techniques: Part 1 of a three-part review series on penile prosthetic urgery. J Sex Med 2009;6:675-81.

7. Levine LA, Becher EF, Bella AJ, et al. Penile prosthesis surgery: Current recommendations from the international consultation on sexual medicine. J Sex Med 2016;13:489-518.

8. Trost L, Wanzek P, Bailey G. A practical overview of considerations for penile prosthesis placement. Nat Rev Urol 2016;13:33-46.

9. Natali A, Olianas R, Fisch M. Penile implantation in Europe: successes and complications with 253 implants in Italy and Germany. J Sex Med 2008;5:1503-12

10. Chierigo F, Capogrosso P, Dehò F, et al. Long-term follow-up after penile prosthesis implantation - survival and quality of life outcomes. J Sex Med 2019;16:1827-33.

11. Kim YD, Yang SO, Lee JK, et al. Usefulness of a malleable penile prosthesis in patients with a spinal cord injury. Int J Urol 2008;15:919-23.

12. Minervini A, Ralph DJ, Pryor JP. Outcome of penile prosthesis implantation for treating erectile dysfunction: experience with 504 procedures. BJU Int 2006;97:129-33.

13. Moses RA, Anderson RE, Kim J, et al. Erectile dysfunction management after failed phosphodiesterase-5-inhibitor trial: A cost-effectiveness analysis. Transl Androl Urol 2019;8:387-94.

14. Li K, Brandes ER, Chang SL, et al. Trends in penile prosthesis implantation and analysis of predictive factors for removal. World J Urol 2019;37:639-46.

15. Kashanian JA, Golan R, Sun T, et al. Trends in penile prosthetics: Influence of patient demographics, surgeon volume, and hospital volume on type of penile prosthesis inserted in New York State. J Sex Med 2018;15:245-50.

16. Bajic P, Mahon J, Faraday M, et al. Etiology of erectile dysfunction and duration of symptoms in patients undergoing penile prosthesis: A systematic review. Sex Med Rev 2020;8:333-37.

17. Menard, J, Tremeaux, J, Faix, A, et al. Erectile function and sexual satisfaction before and after penile prosthesis implantation in radical prostatectomy patients: A comparison with patients with vasculogenic erectile dysfunction. J Sex Med 2011;8:3479-86. 
18. Pillay B, Moon D, Love C, et al. Quality of life, psychological functioning, and treatment satisfaction of men who have undergone penile prosthesis surgery following robotassisted radical prostatectomy. $J$ Sex Med 2017;14:1612-20. 
Figures and Tables

\begin{tabular}{|c|c|}
\hline Variables & \\
\hline \multicolumn{2}{|l|}{ Province in which practicing, $\mathrm{n}(\%)$} \\
\hline British Columbia & $8(23.5)$ \\
\hline Alberta & $4(11.8)$ \\
\hline Saskatchewan & $1(2.9)$ \\
\hline Manitoba & $1(2.9)$ \\
\hline Ontario & $11(32.4)$ \\
\hline Quebec & $2(5.9)$ \\
\hline Nova Scotia & $2(5.9)$ \\
\hline New Brunswick & $4(11.8)$ \\
\hline Newfoundland & $1(2.9)$ \\
\hline \multicolumn{2}{|l|}{ Nature of practice, $\mathrm{n}(\%)$} \\
\hline Academic & $22(64.7)$ \\
\hline Community & $12(35.3)$ \\
\hline \multicolumn{2}{|l|}{ Manufacturer of devices used, $\mathrm{n}(\%)$} \\
\hline American Medical Systems & $30(88.2)$ \\
\hline Coloplast & $0(0)$ \\
\hline+2 & $4(11.8)$ \\
\hline \multicolumn{2}{|l|}{ Number of cases performed per year, $\mathrm{n}(\%)$} \\
\hline 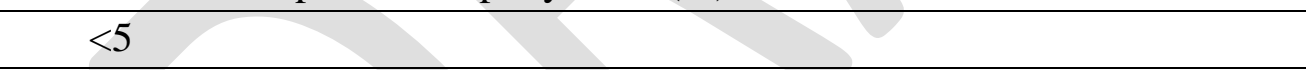 & $10(29.4)$ \\
\hline$+\infty$ & $17(50)$ \\
\hline 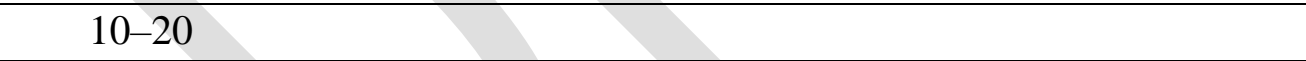 & $0(0)$ \\
\hline $20-30$ & $4(11.8)$ \\
\hline $30-50$ & $2(5.9)$ \\
\hline+3 & $1(2.9)$ \\
\hline Estimated proportion of cases using malleable device, mean \% (range) & $12.6(0-85.0)$ \\
\hline Estimated proportion of cases using IPP, mean \% (range) & $87.4(15.0-100)$ \\
\hline \multicolumn{2}{|l|}{ Coverage for malleable device, $\mathrm{n}(\%)$} \\
\hline Full & $28(82.4)$ \\
\hline Partial & $0(0)$ \\
\hline None & $4(11.8)$ \\
\hline Other & $2(5.9)$ \\
\hline Coverage for IPP, $\mathrm{n}(\%)$ & \\
\hline
\end{tabular}




\begin{tabular}{|l|c|}
\hline Full & $23(67.6)$ \\
\hline Patient pays difference in cost between IPP and malleable & $6(17.6)$ \\
\hline None & $4(11.8)$ \\
\hline Other & $1(2.9)$ \\
\hline Estimated cost in dollars to patient if applicable, median (range) & \\
\hline Malleable, $\mathrm{n}=5$ & $5000(2000-8000)$ \\
\hline IPP, $\mathrm{n}=11$ & $6000(3500-12000)$ \\
\hline
\end{tabular}

